\title{
NOTE
}

\section{Rebuttal to R. J. Miller's comment on Jellett et al. (1989)}

\author{
J. F. Jellett, J. A. Novitsky, J. A. Cantley, R. E. Scheibling \\ Departments of Biology and Microbiology, Dalhousie University, Halifax, Nova Scotia, Canada B3H 4J1
}

In his critique (Miller 1990), Dr Miller is quite right in his first point regarding the mention of sediment sampling in the title, abstract and introduction of our paper (Jellett et al. 1989) and subsequent lack of any information on sediments (other than those collected in the aquarium) in the main body of the paper. This was an unfortunate oversight which was not detected either by ourselves or by the reviewers of the manuscript and we regret any confusion this may have caused. Sediment samples were taken during the course of this study at Eastern Passage, at the Outfall Site, and at 30 and $60 \mathrm{~m}$ from the outfall. One $\mathrm{ml}$ subsamples were plated and examined for amoebae as for the filters. All results were negative and we decided that this avenue was not worth pursuing. This information should have been stated in the paper but was accidentally deleted in an earlier draft.

Dr Miller questioned the adequacy of our sample sizes. Certainly, we would have liked to survey more sea urchins for paramoebiasis, use more cages, isolate more amoebae and conduct more infection experiments. However, our resources were limited in this preliminary study and we had to make certain choices. We feel that 100 sea urchins taken at random from a heterogenous population within Halifax Harbour at the time of the seawater temperature minimum represents an adequate sample size to survey for latent paramoebiasis. If the amoebae are able to overwinter in Strongylocentrotus droebachiensis, we are confident that we would have been able to detect their presence in the 5 radial nerve and coelomic fluid samples that were taken from each of these 100 specimens, and that a further sampling of this population at another time during the winter months would not have been more fruitful. Subsequent screenings of sea urchins from Halifax Harbour at other times during winter (unpubl.) are consistent with our reported results - we have never recovered amoebae from sea urchins at temperatures suboptimal for paramoebiasis. However, we do clearly state in our paper that we have not ruled out the possibility of other hosts or habitats for this organism. Individuals used in our caging study were healthy' (rather than 'apparently healthy'). We determine the health of sea urchins used in all our disease experiments by holding them at disease temperatures for at least $3 \mathrm{wk}$ prior to exposure to disease. This is quite important in terms of control of our experiments and we clearly make a distinction in our paper between healthy (used in the caging and infection experiments) and apparently healthy (collected from Bedford Basin for dissection) sea urchins. We had to make decisions on how best to use a limited number of large (and expensive) cages in our biological experiments. Moreover, we had limited time available with seawater temperatures in the range required for transmission of the disease, to conduct the experiments. This was a preliminary experiment designed to detect the release of pathogenic amoebae from the Aquatron and was clearly stated as such in the paper. The single amoeba that we isolated represents a random sample drawn from a population near the outfall. Although we would have liked to have isolated more amoebae and done more infection experiments, we were limited by the time-consuming and expensive nature of these experiments (each required a $30 \mathrm{~d}$ experimental exposure in our temperature-controlled, running-seawater infection apparatus, followed by dissection of the 66 sea urchins involved, followed by daily microscopic examinations of the 66 coelomic fluid samples and the 330 radial nerve samples for $3 \mathrm{wk}$ ).

We believe that our conclusions are clearly stated and logically sound. Since the infection of the caged animals was sporadic, we conclude that although 'virulent Paramoeba invadens is released from the Aquatron outfall and can cause disease in caged sea urchins... Two possibilities exist which could explain the sporadic infection... (1) virulent amoebae are not released in sufficient quantity (through dilution in the 
outfall pipe and on subsequent release), or (2) some of those that are released have lost virulence'. We go on to support the second possibility through the isolation of avirulent $P$. invadens from the outfall. At no time do we state that all the amoebae released are either virulent or avirulent, and we do not feel that our results are contradictory at all.

We do not claim to have conclusively demonstrated that the causative agent of sea urchin disease, Paramoeba invadens, is not endemic to the Atlantic coast of Nova Scotia. Our study is the first and only attempt to recover this amoeba from the natural environment. Clearly, we were limited in terms of the number of locations and times that we could sample. We were fully aware of our sampling deficiencies (which we have specifically addressed in this rebuttal) and we couched our conclusions appropriately in the paper.

We do not disagree with Dr Miller's comment that our 'conclusions could have benefitted from more thorough research'. These are preliminary findings and for this reason we submitted the work as a note. We felt (and the reviewers obviously agreed) that our results warranted publication, even in their preliminary form. Our hope is that our study may stimulate other workers (such as Dr Miller) to extend the work, to refine the hypotheses and approaches, and thereby add to the state of knowledge.

Lastly, regarding Dr Miller's concern that we did not adequately refer to his work, we maintain that we have used the most appropriate references in all cases. We regret that Dr Miller differs in his opinion on this point.

We welcome healthy and constructive criticism, especially when it illuminates serious flaws in logic or methodology that may misdirect workers, or when it provides alternative hypotheses or interpretations of phenomena that can bolster our understanding of a particular problem. Dr Miller's critique does neither of these things. We do, however, thank Dr Miller for presenting his difference of opinion, and urge him to take up the challenge to further our preliminary findings.

\section{LITERATURE CITED}

Jellett, J. F., Novitsky, J. A., Cantley, J. A., Scheibling, R. E. (1989). Non-occurrence of free-living Paramoeba invadens in water and sediments of Halifax Harbour, Nova Scotia, Canada. Mar. Ecol. Prog. Ser. 56: 205-209

Miller, R. J. (1990). Comment on Jellett et al. (1989). Mar. Ecol Prog. Ser 63: 305-306

Manuscript received: January 29, 1990 